\title{
Tablet Friability
}

National Cancer Institute

\section{Source}

National Cancer Institute. Tablet Friability. NCI Thesaurus. Code C133975.

The percent loss of a tablet due to mechanical action that results in fracture or breaking during the test. 